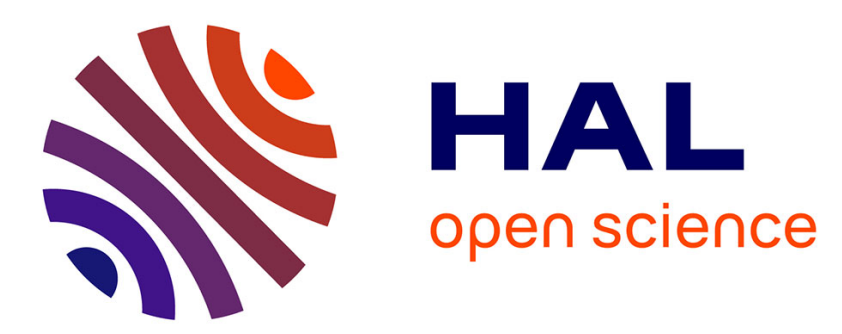

\title{
Resource Sharing in 5G Multi-Operator Wireless Network
}

\author{
Soha Farhat, Abed Ellatif Samhat, Samer Lahoud, Bernard Cousin
}

\section{To cite this version:}

Soha Farhat, Abed Ellatif Samhat, Samer Lahoud, Bernard Cousin. Resource Sharing in 5G MultiOperator Wireless Network. 4th International Conference on Computer Science, Computer Engineering, and Education Technologies (CSCEET 2017), Apr 2017, Beyrouth, Lebanon. hal-01712854

\section{HAL Id: hal-01712854 https://hal.science/hal-01712854}

Submitted on 19 Feb 2018

HAL is a multi-disciplinary open access archive for the deposit and dissemination of scientific research documents, whether they are published or not. The documents may come from teaching and research institutions in France or abroad, or from public or private research centers.
L'archive ouverte pluridisciplinaire HAL, est destinée au dépôt et à la diffusion de documents scientifiques de niveau recherche, publiés ou non, émanant des établissements d'enseignement et de recherche français ou étrangers, des laboratoires publics ou privés. 


\title{
Resource Sharing in 5G Multi-Operator Wireless Network
}

\author{
Soha Farhat ${ }^{1,2}$, Abed Ellatif Samhat ${ }^{1}$, Samer Lahoud ${ }^{2}$, Bernard Cousin ${ }^{3}$ \\ soha.farhat@ul.edu.lb, samhat@ul.edu.lb, samer.lahoud@usj.edu.lb, bcousin@irisa.fr \\ ${ }^{1}$ Lebanese University- CRSI, Lebanon, ${ }^{2}$ University of Saint Josef -ESIB, Lebanon \\ ${ }^{3}$ University of Rennes 1 -IRISA, France
}

\begin{abstract}
In a multi-operator shared network, an appropriate decision is needed for how much to share resources in order to maintain user satisfaction, while maximizing cooperation benefits. In this paper we investigate resource sharing in a three operators sharing system. The study focuses on the value of the sharing factor, which is the percentage of the operator resources dedicated for guest users access. Simulation results showed that resource reservation improves client satisfaction in terms of blocking rates, and the value of the sharing factor affects the achieved profits of the sharing operators. Besides, the best decision of the sharing factor and the pricing scenario depends on the capacity of the operator.
\end{abstract}

Index Terms-5G; multi-operator wireless networks; resource sharing; inter-operator service pricing; user satisfaction; operator profits.

\section{INTRODUCTION}

The fifth generation of mobile networks (5G), is expected to provide a much greater capacity than the fourth generation $(4 \mathrm{G})$, in order to meet the growing demand. The $5 \mathrm{G}$ network will be able to provide very high data rates, with energy efficiency [1], [2]. Different technologies will complement each other to achieve the objectives of 5G [1], [3]. Moreover, software solutions will be essential, in $5 \mathrm{G}$, for a faster deployment of new network features [4], [5]. Some regulators as ARCEP finds that mobile network sharing agreements can accelerate and reduce deployment costs [6]. The mobile network sharing involves sharing between multiple operators part or all of the equipment constituting their mobile networks. In our model, we consider a multi-operator system, where a mobile user can be served through the network of his home operator, or it will be transferred and served through the network of another operator. The latter will determine the service cost, to be paid by the home operator of the transferred user. In previous works [7], [8], [9], we proposed an algorithm for the access selection decision, and we showed its efficiency compared to other selection algorithms using MADM (Multiple Attribute Decision Making) methods. The previous study was made in a sharing system without making any resource reservation and results showed that, with an open access mode, the operator having the highest capacity was penalized by serving a high number of guest users.

In this paper, we propose that cooperating operators will share a limited amount of their resources. Our study focuses on the sharing factor, which is the percentage of the operator resources dedicated for guest users access. We investigate different values of the sharing factor and we examine how it affects user satisfaction and operators profits.

The remainder of the paper is organized as follows: section 2 presents some existing work related to network sharing and inter-operator service pricing. Section 3 describes our the system model. Simulation environment and results are presented in section 4. Finally, conclusion is made in section 5 .

\section{BACKGROUND AND RELATED WORKS}

In a multi-operator heterogeneous network, a new "flex service" paradigm was introduced in [10]. It allows a mobile user subscribed to "Flex service" to dynamically access base stations (BSs) of different providers. Authors present two modeling framework for the access at both microscopic and macroscopic levels. At a microscopic level, a flex user accesses dynamically base stations of different providers based on various criteria, such as profile, network conditions and offered prices. In our work, we envisage a similar multi-operator environment, but mobile user does not need any previous subscription and the access selection decision is controlled by the home operator. In multi-operator network, the majority of the works use game theory for the access selection and joint inter-operator service pricing. In [11], authors consider that part of the BS infrastructure may be switched off during low traffic conditions, motivating MNOs to share the resources of the remaining active BSs in the same cell. Another model of base station sharing is presented in [12], where authors used a game theoretic formulation to decide about turning on or off each BS in order to maximize the global utility. In a previous work [13], we proposed three pricing scenarios for the inter-operator service cost, in a multi-operator environment. These scenarios were analyzed and compared to different price sharing scenarios, and the results showed that our pricing scenarios guarantee better profits for the operators sharing high capacity and setting high service price. In [14], we modeled the interaction between wireless operators, in a multi-operator sharing network, as a multi-leader-follower (Stackelberg) game. Cooperating service operators announce their transaction cost in the first stage and the home operator of the transferred user performs the selection decision in the second stage. The game solution is found using Nash equilibrium concept, and the best response is determine for every pairs of leaders. 


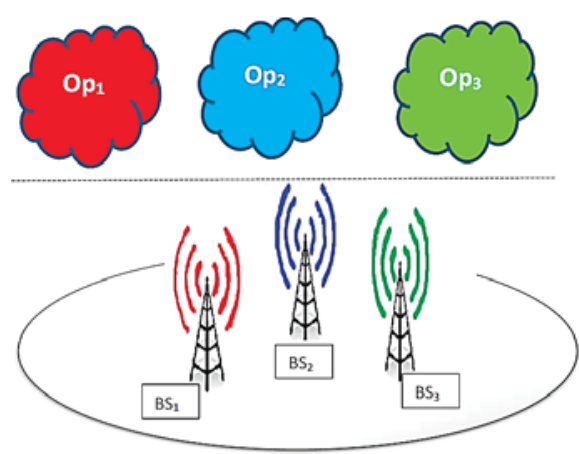

Figure 1: System Model

In this paper, we consider resource sharing using different sharing factor, and we exploit the previously proposed pricing scenarios in a three operators sharing system. We showed the effect of the sharing factor and the pricing scenarios on the user satisfaction and the achieved profits of the sharing operators.

\section{SySTEM MOdEL}

We consider a system formed by three operators, $O p_{1}, O p_{2}$ and $O p_{3}$, each managing a single radio access network fig.1. We suppose that all operators in the system decide to share a fixed percentage and without changing it during the hole period of sharing. And, we use the values 1700, 11000 and $5500 \mathrm{kbps}$ for the capacity of $O p_{1}, O p_{2}$ and $O p_{3}$, respectively. We track the variation of the blocking rates, as well as the achieved profits, for different values of the sharing factor $\gamma$, considering the values $\gamma=10,20,30$ and $50 \%$.

Besides, each operator sets a price $p$ for its client, we used the values $0.9,0.1$ and 0.2 units/kbytes for the prices of $O p_{1}, O p_{2}$ and $O p_{3}$, respectively. In the case of transferring a user, the price $p$ is always paid to the home operator and the latter will pay a service cost $C s$ set by the new service operator. The service cost $C s$ is set according to the adopted pricing scenario. We consider three pricing scenarios previously proposed in [13]:

1) ACAG (As Client As Guest): each operator of the sharing system sets its service cost $C s$ equal to its price $p$ set for its own clients, such that $C s_{i}=p_{i}$ for all operator $_{i} i=1,2$ and 3.

2) MIWC (Max In When Cooperating): all operators set identical $C s$ equal to the highest price $p$ in the system, such that $C s=\max _{i}\left(p_{i}\right) i=1,2$ and 3 .

3) MCWC (Min Cost When Cooperating): all operators set identical $C s$ equal to the lowest price $p$ in the system, such that $C s=\min _{i}\left(p_{i}\right) i=1$, 2and 3 .

Further, in order to transfer the user to the suitable operator, we adopt our Nearest Performance and Best Profit Access Selection algorithm NP-BPA [9], that ensures the mobile user and its home operator satisfaction at the same time. It uses a cost function that combines the offered QoS from the radio access networks and the requirements of the mobile user's application, added to the resulting profit from the user transfer. The cost function for the user transfer to operator $i$ is calculated as follows:

$$
S_{i}^{N P-B P A}=W u \cdot\left|S_{u}-S_{i}^{\prime} S A W\right|-W o p \cdot\left(p-C s_{i}\right)
$$

where, $S_{u}$ is the user score and $S_{i}^{\prime} S A W$ is the $\mathrm{i}^{\text {th }}$ service operator score (for more details readers are referred to [9]). The selected operator is the one having the lowest $S_{i}^{N P-B P A}$.

\section{Simulations And Results}

We model the arrival and departure of users as a Poisson Process with mean arrival interval $1 / \lambda s e c$. Once connected, the user will stay in the system for a service time, assumed to follow an exponential distribution of mean $1 / \mu$; we consider a typical value of $1 / \mu=4 \mathrm{~min}$. At the end of the connection, the user will leave the system thus, improving the available bandwidth of the serving operator. The simulation is implemented in MATLAB and the results are given with a confidence interval of $90 \%$. We use the values $1 / \lambda=$ $2.7,3,3.33,4,5,7,9,12,24 \mathrm{sec}$ for the mean inter-arrival interval.

\section{A. Blocking Rate Variation}

In this subsection, we represent the variation of the blocking rates with respect to the sharing factor and for each pricing scenario. Figures 2, 3 and 4 show the blocking rates for $O p_{1}, O p_{2}$ and $O p_{3}$, respectively. The open access case corresponds to the value of $\gamma=100 \%$, high rates.

1) $\mathrm{Op}_{1}$ 's blocking rates: Figures $2 \mathrm{a}, 2 \mathrm{~b}$ and $2 \mathrm{c}$ show the blocking rates for $O p_{1}$ when adopting ACAG, MIWC and MCWC, respectively. It is clear that the blocking rates of $O p_{1}$ decrease when the partners share more capacity, whatever is the adopted pricing scenario. These rates achieve the lowest values with $\gamma=100 \%$ and are high with $\gamma=10 \%$, even at low arrival rates. In fact, $O p_{1}$ has a limited capacity and transfer clients with high rate. Consequently, when partners reduces the amount of shared capacity it reduces the acceptance of guest users, thus, the probability of blocking $O p_{1}$ 's clients increases. Same results are detected when the operators change the pricing scenario from ACAG to MIWC and MCWC.

2) $\mathrm{Op}_{2}$ 's blocking rates: Figures $3 \mathrm{a}, 3 \mathrm{~b}$ and $3 \mathrm{c}$ show the blocking rates for $\mathrm{Op}_{2}$ when adopting ACAG, MIWC and MCWC, respectively. For low arrival rates, below 0.2 , the blocking rates of $\mathrm{Op}_{2}$ with different $\gamma$ and for all pricing scenarios are null. These rates increase with the system arrival rate, i.e. when the system becomes more loaded, and are higher when $\mathrm{Op}_{2}$ shares more capacity with partners. $O p_{2}$ serves high numbers of guest users, thus, with small value of $\gamma$ $(\gamma=10 \%)$, the blocking rates are maintained below $2 \%$, with all pricing scenarios. Hence, reducing the amount of shared capacity helps $O p_{2}$ to limit the guest flow and guarantee its clients satisfaction.

3) $\mathrm{Op}_{3}$ 's blocking rates: Figures $4 \mathrm{a}, 4 \mathrm{~b}$ and $4 \mathrm{c}$ show the blocking rates for $\mathrm{Op}_{3}$ when adopting ACAG, MIWC and MCWC, respectively. Results show the same as for $O p_{1}$; the blocking rates decrease when the partners share more capacity, 
whatever is the adopted pricing scenario. However, for $O p_{3}$ these rates are null at low arrival rates.

We can conclude that the value of $\gamma$ affects the clients satisfaction of all partners. Besides, the operator sharing large capacity and serving a large number of guest users have to reduce the sharing factor in order to guarantee better performance in terms of blocking rates. However, this decision may affect clients satisfaction of other partners having smaller capacity.

\section{B. Global Profits Variation}

In this subsection, we represent the variation of the profits with respect to the adopted pricing scenario and for the values of $\gamma=100,50$ and $10 \%$. Figures 5, 6 and 7 show the achieved profits for $O p_{1}, O p_{2}$ and $O p_{3}$, respectively.

1) $\mathrm{Op}_{1}$ 's global profits: Figures $5 \mathrm{c}, 5 \mathrm{~b}$ and $5 \mathrm{a}$ show the achieved profits for $O p_{1}$ with $\gamma=100,50$ and $10 \%$, respectively. First, notice that when partners reduce the amount of shared resources, the achieved profits of $O p_{1}$ decrease. This operator is sharing the lowest capacity, and its revenue depends strongly on the payment of the transferred users. Therefore, when the partners adopt a low sharing factor $\gamma$, the user blockings of $O p_{1}$ increase, thus, reducing its profits fig. 5c. Second, when the partners adopt ACAG and MCWC the achieved profits of $O p_{1}$ are very closed since the majority of the transferred users of $O p_{1}$ goes to $O p_{2}$, which sets the lowest service price. With MIWC, $O p_{1}$ pays all the users' payment for the transaction cost, and its profit remains the same even when the sharing factor increases. For $O p_{1}$, sharing the lowest capacity, the open access mode, with a low transaction cost pricing scenario guarantees the highest profits.

2) $O p_{2}$ 's global profits: Figures $6 \mathrm{a}, 6 \mathrm{~b}$ and $6 \mathrm{c}$ show the achieved profits for $\mathrm{Op}_{2}$ with $\gamma=100,50$ and 10\%, respectively. This operator is sharing the highest capacity in the system, and its profits improvement depends strongly on the service cost gained from serving guest users. Therefore, when it increases the sharing factor $\gamma, O p_{2}$ serves more guest users and the achieved profits increase. Moreover, MIWC scenario guarantees the highest profits for $\mathrm{Op}_{2}$. And the profits achieved with ACAG and MCWC are the same, since $O p_{2}$ sets the lowest service price. For $\mathrm{Op}_{2}$, sharing the highest capacity, the open access mode guarantees better profits, but with the highest service cost pricing scenario .

3) $\mathrm{Op}_{3}$ 's global profits: Figures $7 \mathrm{a}, 7 \mathrm{~b}$ and $7 \mathrm{c}$ show the achieved profits for $O p_{3}$ with $\gamma=100,50$ and 10\%, respectively. This operator is sharing a medium capacity, it serves guest users coming from $O p_{1}$ at low and medium arrival rates, and transfers its clients to $O p_{2}$, at high arrival rates. Thus, the profit improvement of $\mathrm{Op}_{3}$ depends on the service cost gained from $O p_{1}$ and the income from transferred users at high arrival rates. Therefore, when partners increase the sharing factor $\gamma, O p_{3}$ serves more guest users and its able to transfer more clients, thus, achieving more profits. Moreover, MIWC scenario guarantees the highest profits for $\mathrm{Op}_{3}$ especially at low and medium arrival rates, when it acts as a service operator. But at high arrival rates MIWC induces high service costs, which degrade its profits. This negative

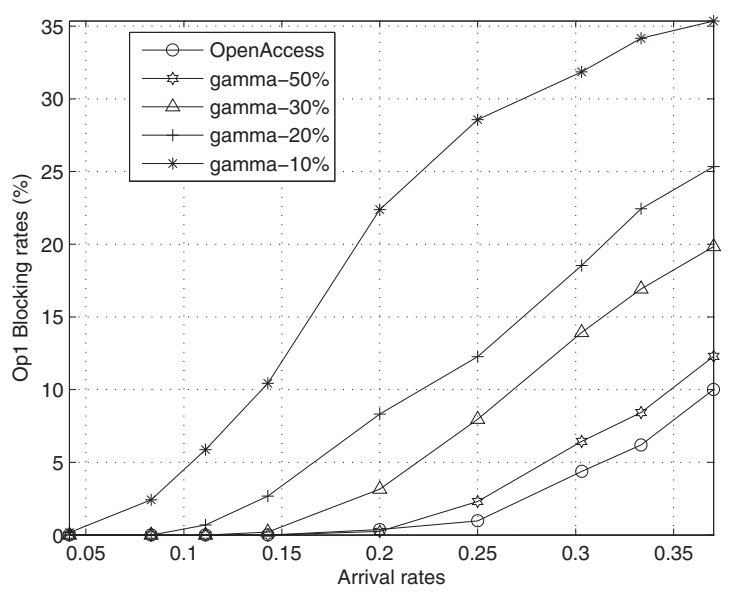

(a) Op1's blocking rates with ACAG

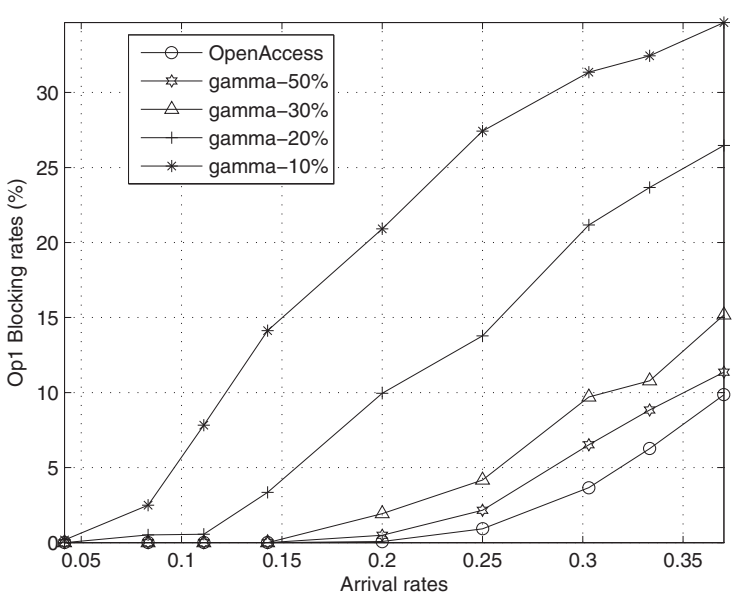

(b) Op1's blocking rates with MIWC

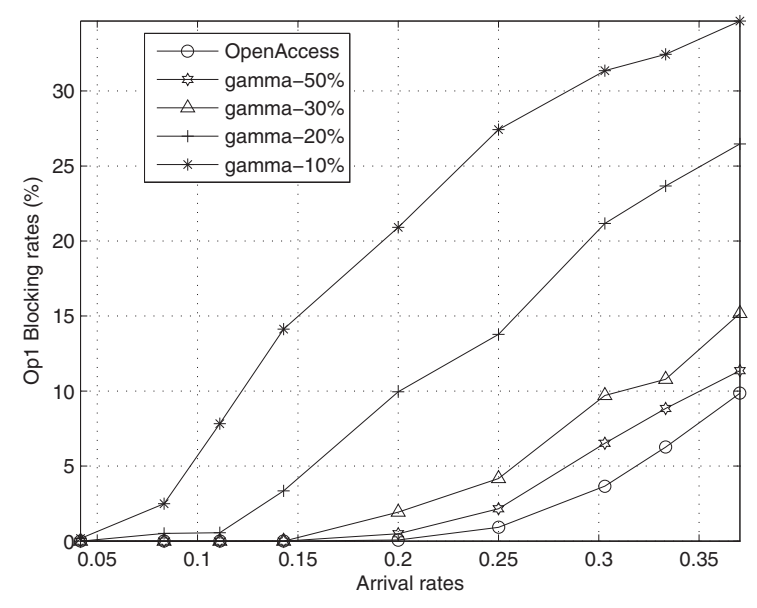

(c) Op1's blocking rates with MCWC

Figure 2: Op1's blocking rates comparison with static sharing 


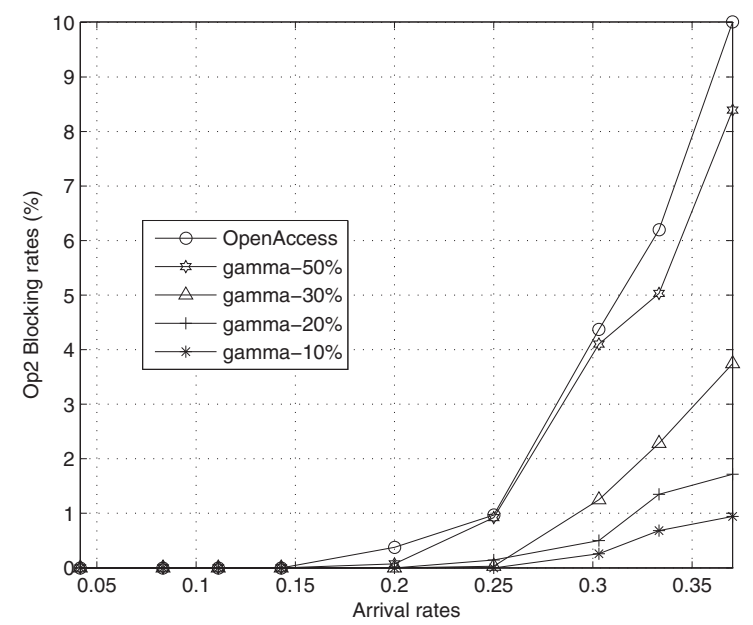

(a) Op2's Blocking rates with ACAG

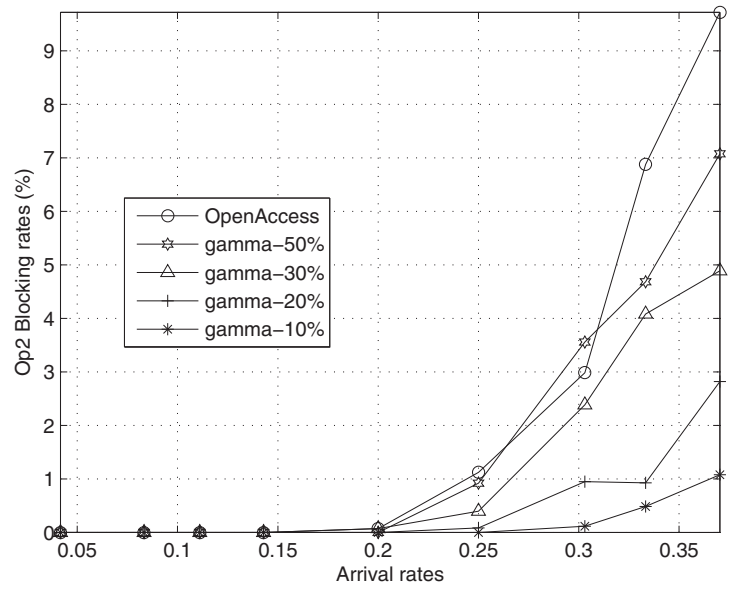

(b) Op2's Blocking rates with MIWC

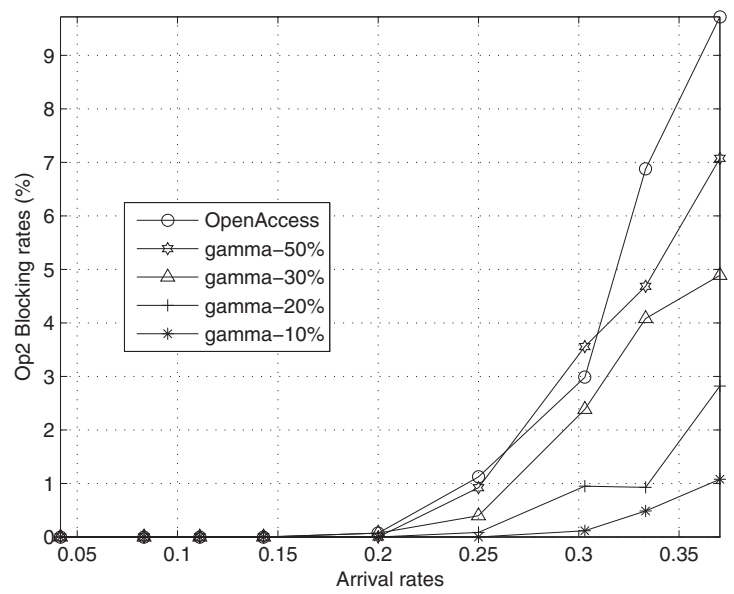

(c) Op2's Blocking rates with MCWC

Figure 3: Op2's Blocking Rates Comparison with static sharing

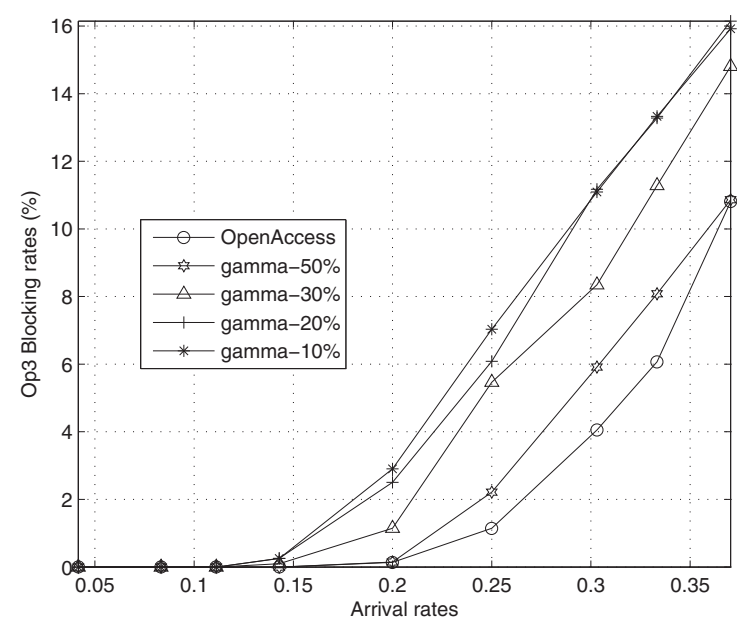

(a) Op3's Blocking rates with ACAG

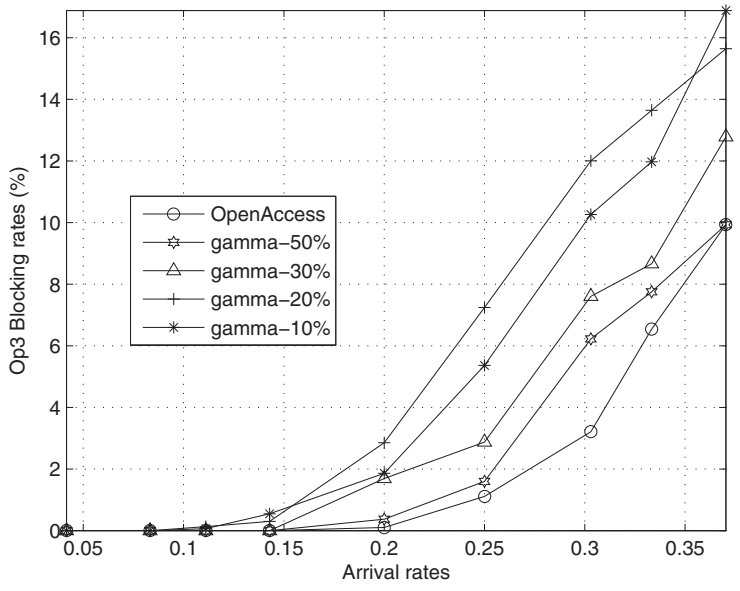

(b) Op3's blocking rates with MIWC

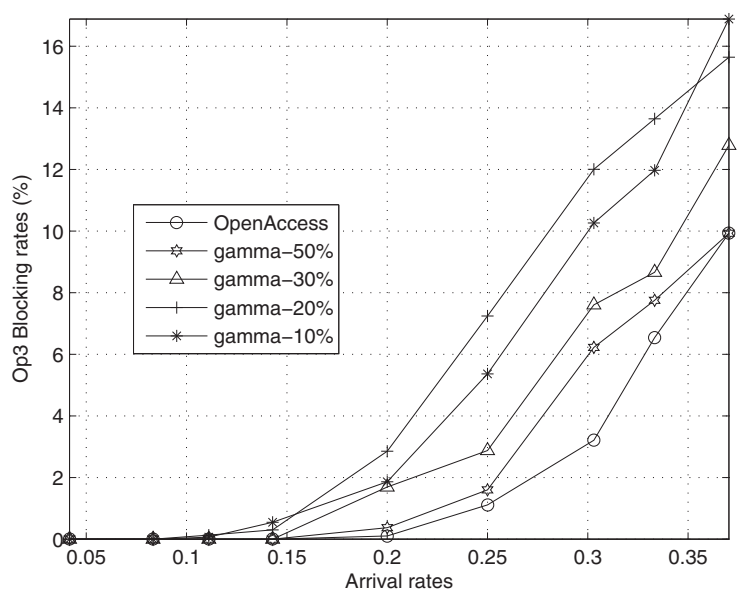

(c) Op3's blocking rates with MCWC

Figure 4: Op3's blocking rates comparison with static sharing 
profit variation decreases with the sharing factor where the profits are smaller.

\section{CONCLUSION}

In this paper we investigated resource sharing in a multioperator wireless network. We considered a system of three operators. These operators agree for a sharing factor, which is the percentage of the resources shared with guest users, and decide the inter-operator service pricing.

Simulation results showed that resource reservation improves the client satisfaction in terms blocking rates, and prevent overload situations for the operator sharing a high capacity. In addition, higher profits are guaranteed when operators share more capacity. An optimal decision is needed for the sharing factor and the inter-operator service cost, in order to maximize the users and operators satisfaction in the same time. Future work will exploit game theory in order to model the interaction between sharing operators in order to determine the best sharing factor and service cost in the sharing system.

\section{REFERENCES}

[1] 5GPPP, "5g vision," 5GPPP, Tech. Rep., 2015.

[2] NGM, "Ngmn 5g whitepaper," Delivrable, February 2015.

[3] L. J. YOUNG, "Telecom experts plot a path to 5g," IEEE Spectrum, 2015.

[4] I. f. C. S. 5G innovation Center, University of Surrey, " $5 \mathrm{~g}$ whitepaper: The flat distributed cloud (fdc) $5 \mathrm{~g}$ architecture revolution," White Paper, January 2016.

[5] A. Checko, H. Christiansen, Y. Yan, L. Scolari, G. Kardaras, M. Berger, and L. Dittmann, "Cloud ran for mobile networks-a technology overview," Communications Surveys Tutorials, IEEE, vol. 17, no. 1, pp. 405-426, Firstquarter 2015.

[6] ARCEP, "Partage de reseaux mmobile: Projet de lignes directrices et analyse preliminaire des contrats existants," Republique Francaise, ARCEP, Tech. Rep., 2016.

[7] S. Farhat, A. E. Samhat, and S. Lahoud, "Hybrid decision algorithm for access selection in multi-operator networks," in Wireless Communications and Networking Conference (WCNC), 2014 IEEE, April 2014, pp. 2414-2419.

[8] S. Farhat, A. Samhat, S. Lahoud, and B. Cousin, "Best operator policy in a heterogeneous wireless network," in e-Technologies and Networks for Development (ICeND), 2014 Third International Conference on, April 2014, pp. 53-57.

[9] S. Farhat, A. E. Samhat, S. Lahoud, and B. Cousin, "Comparison between access selection algorithms in multi-operator wireless networks," in Applied Research in Computer Science and Engineering (ICAR), 2015 International Conference on, Oct 2015, pp. 1-8.

[10] G. Fortetsanakis, M. Papadopouli, G. Karlsson, M. Dramitinos, and E. Yavuz, "To subscribe, or not to subscribe: Modeling and analysis of service paradigms in cellular markets," in Dynamic Spectrum Access Networks (DYSPAN), 2012 IEEE International Symposium on, Oct 2012, pp. $189-200$.

[11] A. Bousia, E. Kartsakli, A. Antonopoulos, L. Alonso, and C. Verikoukis, "Game theoretic infrastructure sharing in multi-operator cellular networks," Vehicular Technology, IEEE Transactions on, vol. PP, no. 99, pp. 1-1, 2015.

[12] B. Leng, P. Mansourifard, and B. Krishnamachari, "Microeconomic analysis of base-station sharing in green cellular networks," in INFOCOM, 2014 Proceedings IEEE, April 2014, pp. 1132-1140.

[13] S. Farhat, A. E. Samhat, S. Lahoud, and B. Cousin, "Pricing strategies in multi-operator heterogeneous wireless networks," in 2015 7th International Conference on New Technologies, Mobility and Security (NTMS), July 2015 , pp. $1-5$.

[14] S. Farhat, Z. Chahine, A. E. Samhat, S. Lahoud, and B. Cousin, "Access selection and joint pricing in multi-operator wireless networks: A stackelberg game," in Digital Information and Communication Technology and its Applications (DICTAP), 2015 Fifth International Conference on, April 2015, pp. 38-43.

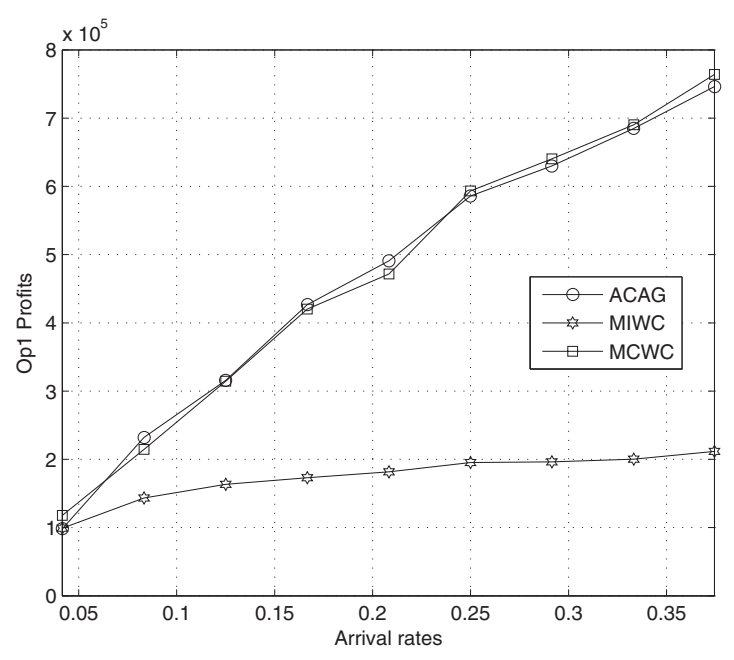

(a) Op1's achieved profits with $\gamma=100 \%$

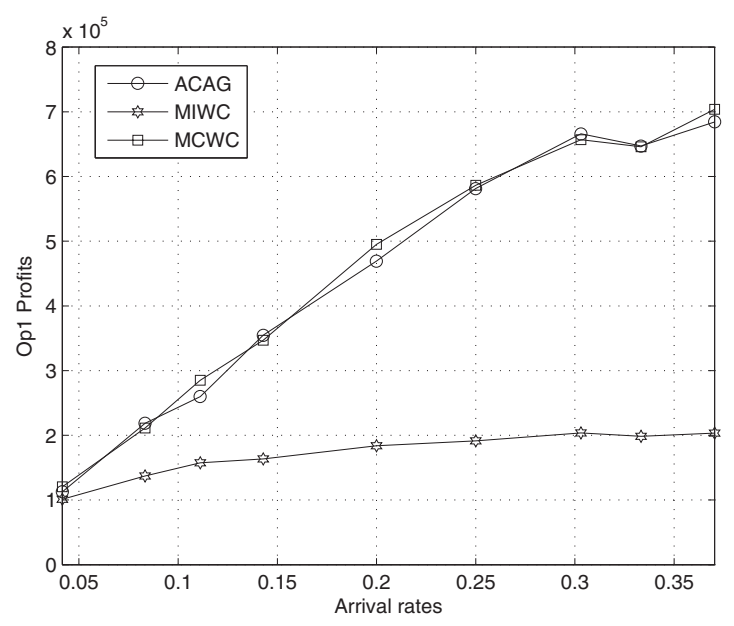

(b) Op1's achieved profits with $\gamma=50 \%$

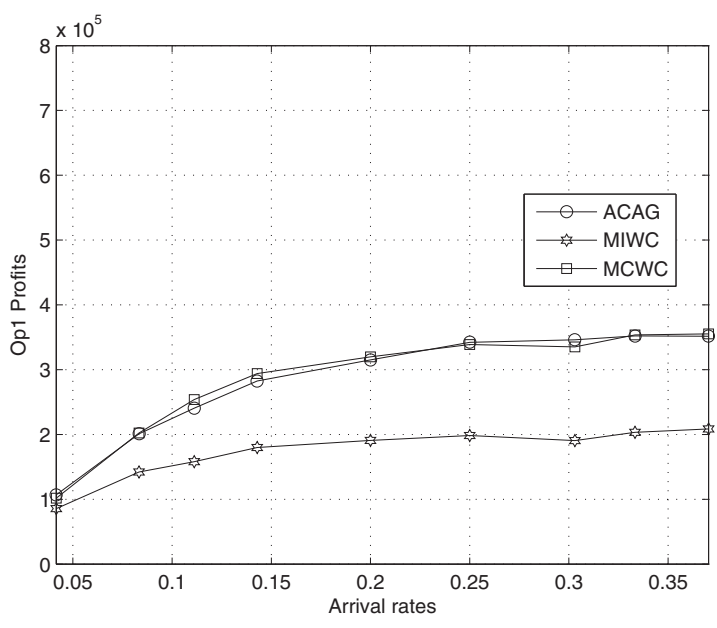

(c) Op1's achieved profits with $\gamma=10 \%$

Figure 5: Opl's achieved profits with static sharing 


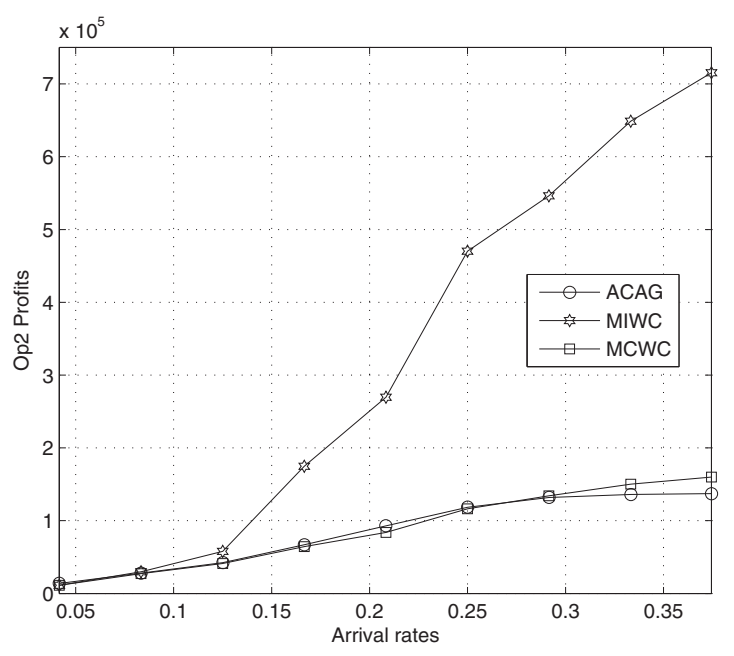

(a) Op2's achieved profits with $\gamma=100 \%$

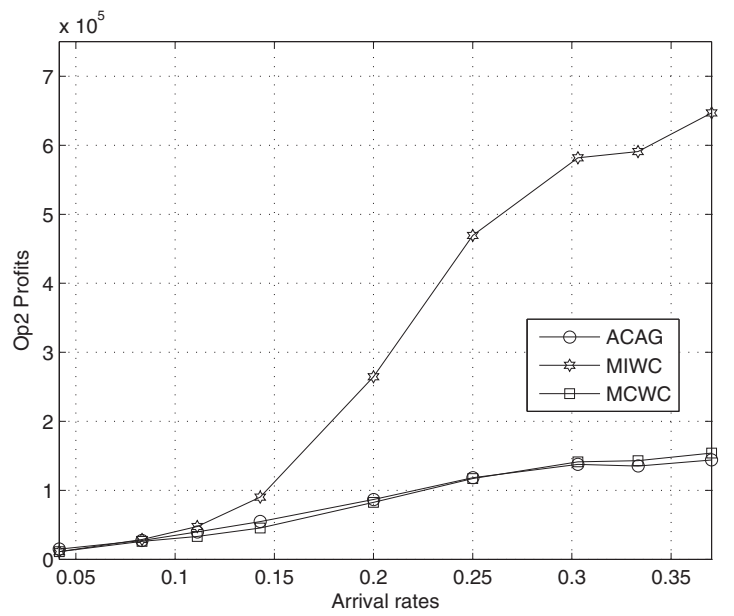

(b) Op2's achieved profits with $\gamma=50 \%$

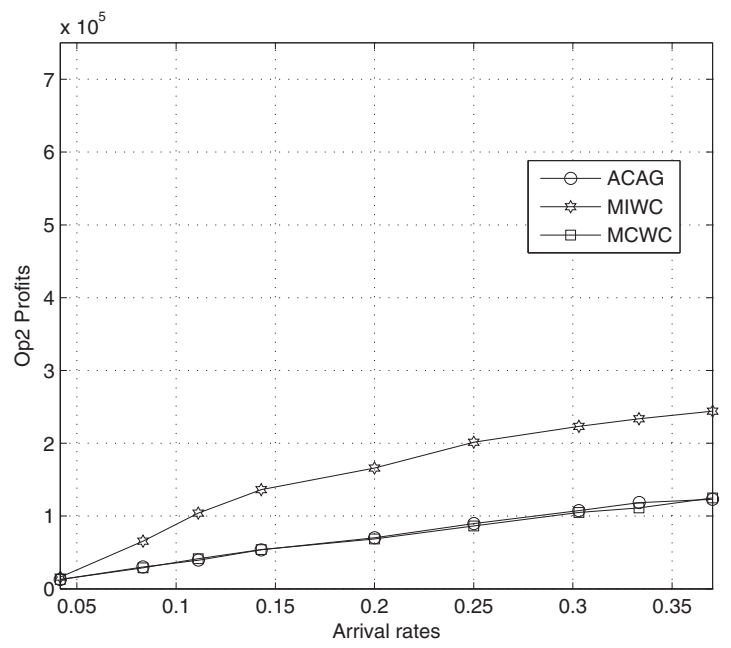

(c) Op2's achieved profits with $\gamma=10 \%$

Figure 6: Effect of the sharing factor and pricing scenario on the achieved profits of the partner sharing the highest capacity, Op2

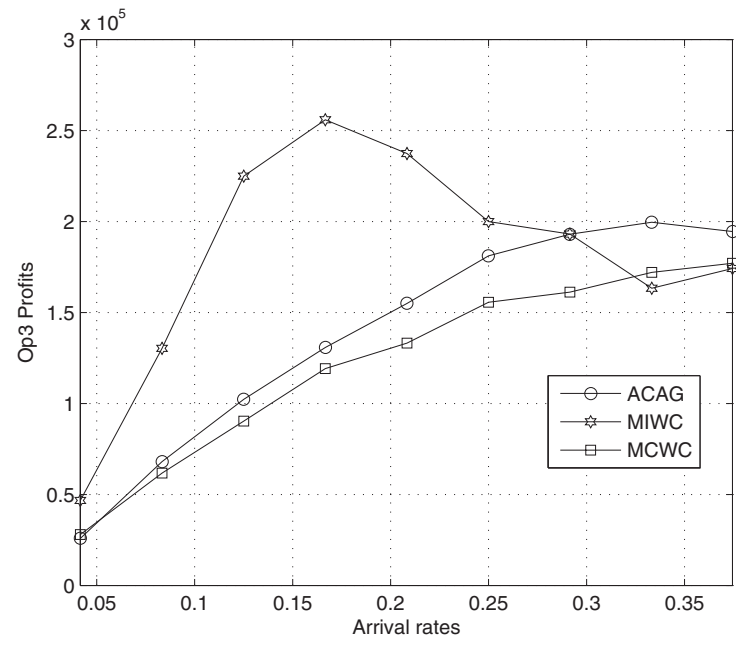

(a) Op3's achieved profits with $\gamma=100 \%$

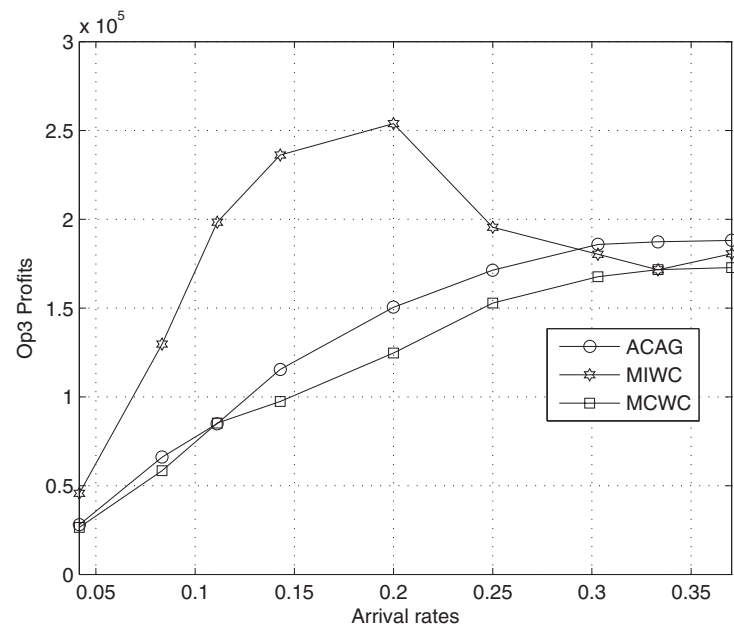

(b) Op3's achieved profits with $\gamma=50 \%$

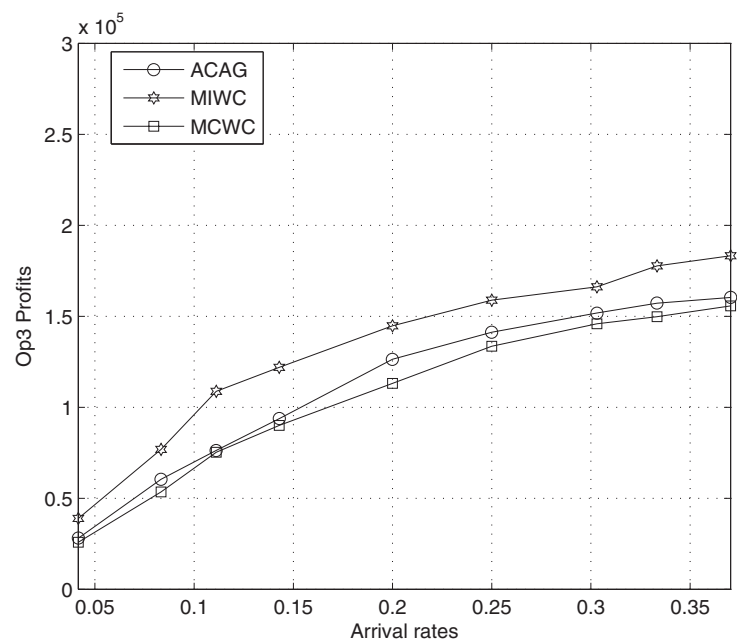

(c) Op3's achieved profits with $\gamma=10 \%$

Figure 7: Effect of the sharing factor and pricing scenario on the achieved profits of Op3 